\title{
Study of the ionization efficiency for nuclear recoils in pure crystals
}

\author{
Y. Sarkis๑, Alexis Aguilar-Arevalo®, and Juan Carlos D’Olivo \\ Instituto de Ciencias Nucleares, Universidad Nacional Autónoma de México, 04510 CDMX, Mexico
}

(Received 22 January 2020; revised manuscript received 6 April 2020; accepted 9 April 2020; published 1 May 2020)

\begin{abstract}
We study the basic integral equation in Lindhard's theory describing the energy given to atomic motion by nuclear recoils in a pure material when the atomic binding energy is taken into account. The numerical solution, which depends only on the slope of the velocity-proportional electronic stopping power and the binding energy, leads to an estimation of the ionization efficiency which is in good agreement with the available experimental measurements for $\mathrm{Si}$ and $\mathrm{Ge}$. In this model, the quenching factor for nuclear recoils features a cutoff at an energy equal to twice the assumed binding energy. We argue that the model is a reasonable approximation for Ge even for energies close to the cutoff, while for $\mathrm{Si}$ is valid up to recoil energies greater than $\sim 500 \mathrm{eV}$.
\end{abstract}

DOI: 10.1103/PhysRevD.101.102001

\section{INTRODUCTION}

In experiments dedicated to the detection of rare events producing low energy depositions ( $\sim 10 \mathrm{keV}$ or less), e.g., direct dark matter (DM) searches or the detection of coherent neutrino-nucleus scattering $(\mathrm{CEN} \nu \mathrm{S})$, the experimental signal generally entails the detection of the recoiling target ions following a scattering event. The amount of electronic excitation produced by a recoiling ion is typically smaller than that produced by a recoiling electron of the same energy, this has been commonly referred to as quenching. The usage of this term may not be in general well justified, since no loss of the elementary electronic excitations (total quanta) is required to occur in order to explain this difference in all cases. Nonetheless, for simplicity and consistency with current literature, hereafter in this work, we will use the term quenching factor $(\mathrm{QF})$ to refer to the ionization efficiency for pure crystals, like $\mathrm{Si}$ and $\mathrm{Ge}$.

In 1963, Lindhard et al. [1] developed a theoretical model that has been used to explain this quenching, aimed at describing energy depositions of the order of a few $\mathrm{keV}$ or higher, when atomic binding energies can be safely neglected. After more than 50 years, the original formulation by Lindhard and collaborators (hereafter referred to as Lindhard's, in short) remains widely in use, and has shown to be successful at describing measurements in this energy regime. As experiments have lowered their detection thresholds reliably observing energy depositions well

Published by the American Physical Society under the terms of the Creative Commons Attribution 4.0 International license. Further distribution of this work must maintain attribution to the author(s) and the published article's title, journal citation, and DOI. Funded by SCOAP. below $1 \mathrm{keV}$, understanding the $\mathrm{QF}$ at those low energies has become crucial to estimate their sensitivities to the physical models they aim to test.

Recent measurements of the QF for nuclear recoils in silicon $(\mathrm{Si})[2,3]$ exhibit a clear deviation from the Lindhard model for energies below $4 \mathrm{keV}$, while data for germanium (Ge) [4-9] are in good agreement.

In a recent article, Sorensen [10] aimed to obtain a $\mathrm{QF}$ valid at lower energies by bringing back the atomic binding energy into Lindhard's original simplified equation. He estimated this binding to be of the order of the electron-hole pair creation energy ( $\sim 3 \mathrm{eV}$ for $\mathrm{Si}$ and $\mathrm{Ge})$, and his solutions exhibit a cutoff of the order of one to a few hundred $\mathrm{eV}$. This result is troublesome [11], since it is not obvious how a low binding energy could produce such a high threshold in the QF. The present work was partially motivated by this observation, and will show that, when properly incorporated into the model, a constant binding energy results in a cutoff in the QF at a value of the same order of magnitude.

This paper is organized as follows. In Sec. II we give a brief summary of the ideas in Lindhard's theory arriving at the simplified integral equation describing the energy given to ions by a recoiling ion in a homogeneous medium, and his equation for the $\mathrm{QF}$, when the binding energy is neglected. In Sec. III we discuss the changes that are needed in order to maintain the binding energy in the model to the lowest order, arriving at a modified version of the simplified integral equation. We propose a simple ansatz for the solution depending on two new parameters, besides the electronic stopping constant $k$ already introduced by Lindhard. We end this section with a description of the numerical solution which depends only on $k$ and the binding energy, which works well in the low energy regime for Ge and $\mathrm{Si}$, most relevant for current and future low-threshold 
DM (e.g., [12-16]) and CELNS (e.g., [17-19]) experiments. In Sec. IV we fit the QF obtained from both, the approximate and numerical solutions, to experimental measurements for $\mathrm{Si}$ and $\mathrm{Ge}$ to find the relevant parameters in each case. The conclusions are presented in Sec. V.

\section{THE LINDHARD MODEL}

When an ion in a homogeneous substance moves with a kinetic energy $E$, heading toward the collision with another ion in the material, after recoiling off an interaction with an incident particle (e.g., the coherent scattering of a neutrino or a DM particle), is sets off a cascade of slowing-down processes that dissipate this energy throughout the medium. If the ion recoils from the interaction with the incident particle with an energy $E_{R}$ and the energy $U$ is lost to disruption of atomic bonding, then $E_{R}=E+U$. Note that, under the assumption of an elastic collision, $E_{R}$ corresponds to the kinetic energy transferred by the incident particle to the struck ion in the material. A sudden approximation is made for the collision, where the timescale of elastic collision is much smaller than timescale of atomic processes. Lindhard's theory [1] concerns with determining the fraction of $E_{R}$ which is given to electrons, $H$, and that which is given to translational motion of ions, $N$, assuming $E_{R}=H+N$. This separation can be written in terms of reduced dimensionless quantities as

$$
\varepsilon_{R}=\eta+\nu,
$$

where $\varepsilon_{R}=c_{Z} E_{R}, \eta=c_{Z} H$, and $\nu=c_{Z} N$, and the scaling factor $c_{Z}=11.5 / Z^{7 / 3} \mathrm{keV}^{-1}$ is defined for a medium with a single atomic species of atomic number $Z$.

The model is simplified by considering the equations obeyed by the average quantities $\bar{\eta}$ and $\bar{\nu}$, for which appropriate probability distributions are assumed to exist, and such that $\varepsilon_{R}=\bar{\eta}+\bar{\nu}$.

It is reasonable to assume that $\bar{\eta}$ represents an upper limit to the available signal in a detector operating in ionization-only mode, such as those used for extreme lowmass WIMP searches and CELNS detection. The nuclear quenching factor is defined as the fraction of the total energy deposited by the incident particle which is transferred to the electrons

$$
f_{n}=\frac{\bar{\eta}}{\varepsilon_{R}}=\frac{\varepsilon+u-\bar{\nu}}{\varepsilon+u}
$$

where $u=c_{Z} U$.

Lindhard considered any physical quantity $\varphi$ (of which $\eta$ and $\nu$ are examples) that is additive over the individual slowing-down processes spawned by the initial scattering. Suppose that a recoiling ion, with kinetic energy $E$, strikes an ion in the medium transferring the energy $T_{n}$ to its center of mass, and the energy $T_{e i}$ to each ionized electron. If $U$, in Lindhard's own words, is the energy spent in "disrupting the atomic binding", then the additivity of $\varphi$ is encoded in the basic integral equation

$$
\begin{aligned}
& \int d \sigma_{n, e}\left[\bar{\varphi}\left(E-T_{n}-\Sigma_{i} T_{e i}\right)+\bar{\varphi}\left(T_{n}-U\right)-\bar{\varphi}(E)\right. \\
& \left.\quad+\Sigma_{i} \bar{\varphi}_{e}\left(T_{e i}-U_{e i}\right)\right]=0
\end{aligned}
$$

where $\sigma_{n, e}$ is the effective cross section for the interaction of the recoiling ion with the ions or electrons in the medium and integration over $\int d \sigma_{n, e}$ represents the sum over all possible interactions (impact parameters). In the last term, $\bar{\varphi}_{e}$ is the function describing the contribution of ejected electrons to $\bar{\varphi}$, each with ionization energy $U_{e i}$. Equation (3) states that the average physical effect caused by the initial recoiling ion before the collision, $\bar{\varphi}(E)$, equals the sum of the average physical effects caused by the ion, the struck ion, and the ejected electrons after the collision. This situation is depicted in Fig. 1. In general, $U$ is not limited to the energy needed to remove the ion from its site, but it can also include contributions to excitation or ionization of bound atomic electrons, and therefore incorporates the Migdal effect [20,21] into the model. In scintillating materials, electronic excitation can be a very significant component of the total signal. In principle, an additional term for the contribution of excited electronic states could be added to Eq. (3), but we will not consider it in the treatment presented here.

Lindhard used five basic approximations in order to cast Eq. (3), for $\bar{\varphi}(E)=\bar{\nu}(E)$, in a simplified form for which he found an approximate numerical solution, expected to be valid for sufficiently large energies: (A) ionized electrons do not produce atomic recoils with appreciable energy, hence the term $\sum_{i} \bar{\varphi}_{e}\left(T_{e i}-U_{e i}\right)$ can
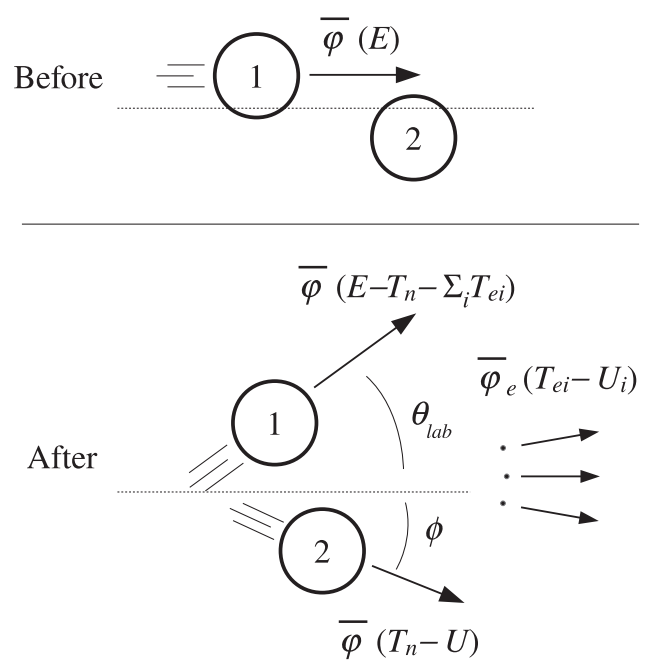

FIG. 1. Scattering of a recoiling ion in the lab frame. The average physical effect of the recoiling ion $\bar{\varphi}(E)$ equals the sum of the average physical effects of the struck ion, the ejected electrons, and itself, after the collision. $U_{i}$ is the ionization energy to free electron $i$. The other quantities are described in the text. 
be dropped; (B) neglect the atomic binding $U$ under the assumption that it is in general smaller than the energy transferred to the recoiling ions, hence $\varepsilon_{R} \approx \varepsilon ;(\mathrm{C})$ the energy transferred to ionized electrons is also small compared to that transferred to recoiling ions; (D) the effects of electronic and atomic collisions can be treated separately; (E) $T_{n}$ is also small compared to the energy $E$.

The interactions between recoiling ions are modeled as two-body elastic scatterings of identical particles in a screened Coulomb potential $V(r)=\left(e^{2} Z^{2} / r\right) \phi_{0}(r / a)$. Here, $\phi_{0}(r / a)$ is the single atom Thomas-Fermi screening function [22] with a corrected length scale $a=0.8853 a_{0}$ / $\left(Z^{1 / 3} \sqrt{2}\right)$, and $a_{0}$ is the Bohr radius. With this model Lindhard found that the atomic scattering cross section could be written as $d \sigma_{n}=d t f\left(t^{1 / 2}\right) / 2 t^{3 / 2}$, where $t=$ $\varepsilon^{2} \sin ^{2}(\theta / 2), \theta$ is the scattering angle in the center of mass, and $f\left(t^{1 / 2}\right)$ is a function only of $t$.

The electronic stopping power can be expressed as $1 / N_{e}(d E / d R)_{e}=\int d \sigma_{e}\left(\Sigma_{i} T_{e i}\right)$ [23], where $N_{e}$ is the electron number density and $R$ is the distance travelled by an ionizing projectile. It appears naturally as a consequence of approximations (C) and (D), and in terms of the reduced quantities $\varepsilon$ and $\rho=\pi a^{2} N_{e} R$, can be written as

$$
S_{e}(\varepsilon)=d \varepsilon / d \rho=k \varepsilon^{1 / 2},
$$

where $k=0.133 Z^{2 / 3} A^{-1 / 2}$. This velocity proportionality of the electronic stopping power appears to hold in a variety of substances, from gaseous to semiconductor targets, although indications of a threshold velocity below which a projectile loses no energy to electrons are known to exist [24].

Putting all these approximations together, including $u=0$ (approximation B), Lindhard arrived at his simplified integral equation for the average energy given to atomic motion

$k \varepsilon^{1 / 2} \bar{\nu}^{\prime}(\varepsilon)=\int_{0}^{\varepsilon^{2}} d t \frac{f\left(t^{1 / 2}\right)}{2 t^{3 / 2}} \times[\bar{\nu}(\varepsilon-t / \varepsilon)+\bar{\nu}(t / \varepsilon)-\bar{\nu}(\varepsilon)]$.

He found an approximate numerical solution of Eq. (5) imposing the boundary condition that $\bar{\nu}(\varepsilon) \rightarrow \varepsilon$ when $\varepsilon \rightarrow 0$ (and noting that $\bar{\nu}^{\prime \prime}(\varepsilon)<0$ ), from where the $\mathrm{QF}$ in Eq. (2) can be calculated as

$$
f_{n} \approx \frac{\bar{\eta}(\varepsilon)}{\varepsilon}=\frac{\varepsilon-\bar{\nu}(\varepsilon)}{\varepsilon},
$$

which he parametrized in the following way

$$
\begin{aligned}
\bar{\nu}_{L}(\varepsilon) & =\frac{\varepsilon}{1+k g(\varepsilon)}, \quad \bar{\nu}(\varepsilon) \equiv \bar{\nu}_{L}(\varepsilon) \\
g(\varepsilon) & =3 \varepsilon^{0.15}+0.7 \varepsilon^{0.6}+\varepsilon .
\end{aligned}
$$

The last expression is well known to the experimental community studying low energy depositions by nuclear recoils.

It is interesting to note that there is an inconsistency with the boundary condition imposed by Lindhard which, on one hand implies that $\bar{\nu}_{L}^{\prime}(\varepsilon) \rightarrow 1$ when $\varepsilon \rightarrow 0$, as stated above, while on the other, by applying L'Hopital's rule directly to Eq. (5) it can be shown that $\lim _{\varepsilon \rightarrow 0} \bar{\nu}_{L}^{\prime}(\varepsilon)=0$, hinting at the existence of a discontinuity in the first derivative at zero. Despite its limitations, Lindhard's model has been very successful in describing the QF for nuclear recoils in Si up to $\varepsilon \gtrsim 0.1$ (4 keV), and so far all available data for $\mathrm{Ge}$, corresponding to $\varepsilon \gtrsim 0.00088(250 \mathrm{eV})$.

\section{SIMPLIFIED INTEGRAL EQUATION WITH BINDING ENERGY}

We wish to find a version of the simplified integral equation, Eq. (5), where approximation (B) has been removed in a mathematically consistent way. In Ref. [10] this approximation was relaxed by replacing the term $\bar{\nu}(t / \varepsilon)$ with $\bar{\nu}(t / \varepsilon-u)$. While this is certainly part of the required modifications, attention must be paid to the lower limit of integration on the right-hand side of Eq. (5), which should be set to $\varepsilon u$, as is suggested by not allowing the argument of $\bar{\nu}(t / \varepsilon-u)$ to become negative. The same lower limit can be recovered by modeling the atomic scattering as the collision of semihard spheres, as is shown in Appendix A.

In addition to bringing back the binding energy, in going from Eq. (3) to Eq. (5), the term $\bar{\varphi}\left(E-T_{n}-\Sigma_{i} T_{e i}\right)$ has been expanded to first order in $\Sigma_{i} T_{e i} /\left(E-T_{n}\right) \ll 1$, but, it has also been assumed that $T_{n} / E$ is small to some extent (approximation E). In the interest of finding a solution valid for lower energies (e.g., $\varepsilon>0.01$ in $\mathrm{Si}$ ) we will perform a similar expansion, but keeping a term of order $T_{n}\left(\Sigma_{i} T_{e i}\right)$, namely

$$
\begin{gathered}
\bar{\nu}\left(E-T_{n}-\Sigma_{i} T_{e i}\right) \approx \bar{\nu}\left(E-T_{n}\right)-\bar{\nu}^{\prime}(E)\left(\Sigma_{i} T_{e i}\right) \\
+\bar{\nu}^{\prime \prime}(E) T_{n}\left(\Sigma_{i} T_{e i}\right),
\end{gathered}
$$

where terms of order $\left(\Sigma_{i} T_{e i}\right)^{2}$ or higher, have been dropped. The additional term proportional to $\bar{\nu}^{\prime \prime}(E)$ will have an important effect when assessing the accuracy of our approximate solution, and will be key to the implementation of the numerical solution. Substituting Eq. (8) into Eq. (3), and integrating over the nuclear and electronic cross sections, putting also in effect approximation (D), we arrive at our proposed form of the modified simplified integral equation

$$
\begin{aligned}
& k \varepsilon^{1 / 2} \bar{\nu}^{\prime}(\varepsilon)-\frac{1}{2} k \varepsilon^{3 / 2} \bar{\nu}^{\prime \prime}(\varepsilon) \\
& \quad=\int_{\varepsilon u}^{\varepsilon^{2}} d t \frac{f\left(t^{1 / 2}\right)}{2 t^{3 / 2}} \times[\bar{\nu}(\varepsilon-t / \varepsilon)+\bar{\nu}(t / \varepsilon-u)-\bar{\nu}(\varepsilon)],
\end{aligned}
$$


where we have considered a mean value of the energy transferred to the struck ion $\bar{t}_{n} \approx\left\langle t_{n}\right\rangle=\left\langle\varepsilon \sin ^{2} \theta / 2\right\rangle=\frac{1}{2} \varepsilon$, (where $t_{n}=c_{Z} T_{n}=t / \varepsilon$ ) in order to recover the electronic stopping power from the integration of the second order term (see Appendix B).

In what follows we will use the "average" Molière-like screening function given in [23] for the determination of $f\left(t^{1 / 2}\right)$. Other screening functions are available [25,26], and the differences between them can affect the determination of the constant $k$, but are expected to be constrained to the interval $0.1-0.2$.

The model depicted in Fig. 1 requires that prior to producing any effect the struck ion must recoil with a kinetic energy larger than $U$, otherwise the argument in $\bar{\varphi}\left(T_{n}-U\right)$ becomes negative. Modeling the process as the collision of semi-hard spheres (see Appendix A), we recognize $U$ as the depth of the soft part of the potential, and can be associated with the energy given to the electrons occupying the shells above the inner noblelike nonvalence shells of the ion. If sufficient energy is available the collision can induce excitation of electrons from these shells, as well as from the valence to the conduction band, producing a number of electron-hole $(e-h)$ pairs, and possibly also create a vacancy and self-intersticial (Frenkel) pair $[27,28]$ in the lattice. In general $U$ will depend on the kinetic energy of the recoiling ion $E$.

Table I shows the values of the binding energies, relative to the top of the valence band, for electrons occupying inner shells above the $[\mathrm{Ne}]^{2}$ or $[\mathrm{Ar}]^{18}$ cores in $\mathrm{Si}$ and $\mathrm{Ge}$, respectively $[29,30]$. The table also lists the average $e-h$ production energy and the dislocation energy (average energy to create a FrenkelFig. pair) for each element $[27,28]$. In Si, a recoiling ion (labeled 1 in Fig. 1) moving through the lattice with, say $\varepsilon / c_{Z}=350 \mathrm{eV}$ of kinetic energy, could strike an ion (labeled 2 in Fig. 1) and cause an electron from its $2 p$ shell to reach the conduction band $(100 \mathrm{eV}+\mathrm{a}$ fraction of $3.7 \mathrm{eV})$, in addition to causing a handful more to reach it from the valence band. Depending on the number of excited electrons and their energies, the struck ion could also become dislocated from the lattice. Similarly, in Ge, an ion moving with $\varepsilon / c_{Z}=50 \mathrm{eV}$ of kinetic energy could strike an ion and excite an electron

TABLE I. Binding energies, relative to the top of the valence band, for atomic shells between the noble core and the outer valence orbitals [29,30], average $e-h$ creation energies, and dislocation energies $[27,28]$ in $\mathrm{Si}$ and Ge.

\begin{tabular}{|c|c|c|c|c|c|}
\hline \multicolumn{3}{|c|}{ Silicon } & \multicolumn{3}{|c|}{ Germanium } \\
\hline Shell & $U(\mathrm{eV})$ & $\# e$ & Shell & $U(\mathrm{eV})$ & $\# e$ \\
\hline$[\mathrm{Ne}]^{4}$ & & 4 & {$[\mathrm{Ar}]^{18}$} & & $\overline{18}$ \\
\hline $2 p$ & 100 & 6 & $3 d$ & 30 & 10 \\
\hline Average $e-h$ & 3.7 & 4 & Average $e-h$ & 3.0 & 4 \\
\hline Dislocation & 36 & & Dislocation & 23 & \\
\hline
\end{tabular}

from its $3 d$ shell plus a few more from the valence band to the conduction band, or dislocate the ion. Note that the ion that initiates the cascade will also have lost, to atomic processes, some of the recoil energy with which it emerged from the interaction with the incoming particle $\varepsilon_{R}$ (Migdal effect $[20,21])$.

In the remainder of this work we will take $u(\varepsilon)=u$, a constant value, and explore its implications for the QF for nuclear recoils at low energies.

\section{A. Model with a constant $u$}

When $u$ is constant, Eq. (9) is only applicable for $\varepsilon \geq u$, otherwise the lower limit of integration derived from the semi-hard sphere model becomes ill-defined (see Appendix A). Furthermore, since the right-hand side (r.h.s.) of Eq. (9) is the contribution to $\bar{\nu}$ from the recoiling ion (labeled 1 in Fig. 1), it must be non-negative for any $\varepsilon \geq u$. Defining the quantity in square brackets in the integrand as

$$
I(\varepsilon, t)=\bar{\nu}(\varepsilon-t / \varepsilon)+\bar{\nu}(t / \varepsilon-u)-\bar{\nu}(\varepsilon),
$$

two observations are in order: (1) at $\varepsilon=u$ the r.h.s. of Eq. (9) is equal to zero, since the upper and lower limits of integration are equal, therefore, $I(\varepsilon, t) \geq 0$ (must be nonnegative) for any $\varepsilon \geq u$, and (2) evaluating the r.h.s. at any value of $\varepsilon>u$ requires knowledge of the function $\bar{\nu}(\varepsilon)$ for values of $\varepsilon<u$. Note that observation (1) further implies that $\bar{\nu}(\varepsilon)$ has the following linear form in the region below $u$

$$
\bar{\nu}(\varepsilon)=\varepsilon+u=\varepsilon_{R}, \quad \text { for } \varepsilon \leq u .
$$

We now use Eq. (2) to calculate the QF with $\bar{\nu}(\varepsilon)$ as the solution to the problem posed in Eq. (9). From the requirement in Eq. (11), it is clear that the QF will vanish for $\varepsilon \leq u$, or equivalently, for $\varepsilon_{R} \leq 2 u$. In the limit $u=0$ we recover Lindhard's model and QF. The constant $u$ model is one in which no energy will go into the motion of ions unless the initial ion recoils with an energy $\varepsilon_{R}>2 u$.

From the values in Table I we can expect that this model will produce a cutoff in the $\mathrm{QF}$ for $\mathrm{Si}$ at recoil energies of the order of $200-400 \mathrm{eV}$, while for Ge it can be expected at energies of the order of 30-60 eV.

\section{B. Interpolation from low to high $\varepsilon$ behavior}

It has been noted by some authors $[10,11]$ that in Lindhard's original model the energy transferred to electrons is slightly overestimated. This is so because it primarily originates from the electronic stopping power of ions, assumed to be given by Eq. (4), which vanishes at $\varepsilon=0$. However, if we consider that the effect of the binding energy is to suppress the energy transferred to electrons when the recoiling ion has energies below $u$, we can argue that $\bar{\eta}$ needs to be corrected by a certain amount. If the correction is taken to be proportional to the electronic 
stopping power at energy $\varepsilon$ itself, plus a possible offset, we can write

$$
\bar{\eta}=\bar{\eta}_{L}-C_{0}(d \varepsilon / d \rho)-C_{1},
$$

where $\bar{\eta}_{L}$ is the average energy transferred to electrons according to the Lindhard model. Since $\varepsilon=\bar{\eta}_{L}+\bar{\nu}_{L}$, the corrected average energy transferred to atomic motion is

$$
\bar{\nu}=\bar{\nu}_{L}+C_{0} \varepsilon^{\frac{1}{2}}+C_{1}+u,
$$

Notice that the model used in [10] is equivalent to correcting $\bar{\eta}$ by a constant value, however, it is tested against Lindhard's basic integral equation, Eq. (5). The general form in Eq. (13) can be made to approximately follow the required linear behavior expected near and below $u$, posited in Eq. (11), while at the same time coincide with Lindhard's solution at high $\varepsilon$, as can be seen in Fig. 2. Such solution will produce a cutoff in the QF defined in Eq. (2) at $\varepsilon=u$, provided that $\bar{\nu}(u)=2 u$, and that $\bar{\nu}(\varepsilon)>\varepsilon+u$ for $\varepsilon<u$. One could also device a solution for $\bar{\nu}(\varepsilon)$ that equals $\varepsilon+u$ once $\varepsilon$ falls below $u$ by allowing it to have a discontinuity on the first derivative (a kink) at this value.

As a way to measure the quality of our proposed solution we will follow [10] and define the error

$$
\text { Error }=\left|\frac{\text { r.h.s }-1 . h . s}{\text { r.h.s }+ \text { l.h.s }}\right| \text {, }
$$

comparing the left-hand-side (1.h.s) and the right-hand-side (r.h.s.) of the modified integral equation, Eq. (9). As noted

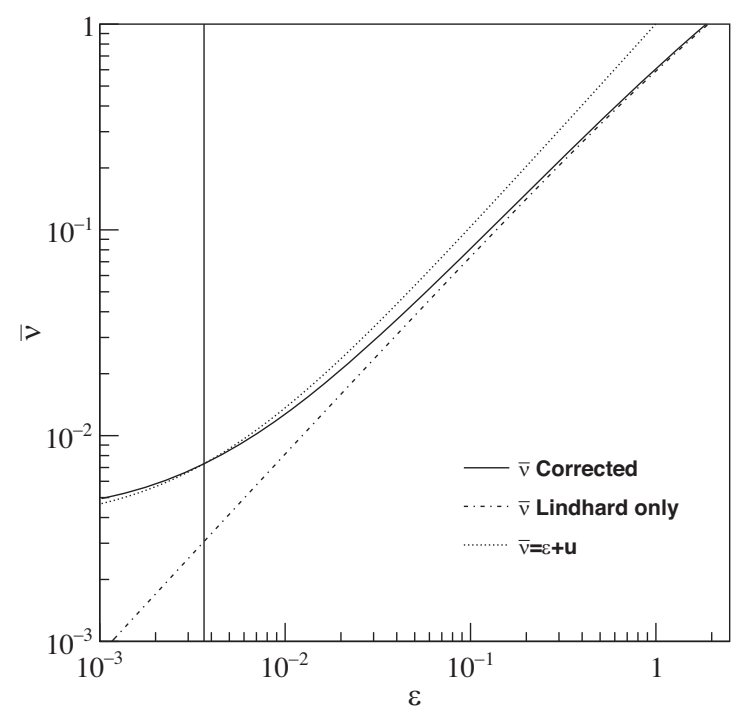

FIG. 2. The function $\bar{\nu}(\varepsilon)$ from Eq. (13) fitted to the $\mathrm{Si}$ experimental data interpolates between the Lindhard solution at high energies, and the expected $\varepsilon+u$ (approximately) below $u$. A cutoff in the QF occurs at the crossing between $\bar{\nu}(\varepsilon)$ and $\varepsilon+u$ at $\varepsilon=u$ (vertical line). in [10] evaluation of the r.h.s. requires knowledge of the function $f\left(t^{1 / 2}\right)$ to lower energies than considered by Lindhard. Therefore, we follow the useful prescription given therein and use the parametrization for the reduced nuclear stopping power $S_{n}(\varepsilon)$, Eq. (15) of [23], to calculate $f\left(t^{1 / 2}\right)$ by differentiation of $\varepsilon S_{n}(\varepsilon)$.

\section{Numerical solution}

From the observations in Sec. III A we write the solution in the form:

$$
\bar{\nu}(\varepsilon)= \begin{cases}\varepsilon+u, & \varepsilon<u, \\ \varepsilon+u-\lambda(\varepsilon), & \varepsilon \geq u\end{cases}
$$

where $\lambda(\varepsilon)$ is a continuous function satisfying $\lambda(u)=0$. In order for Eq. (15) to be a solution to the integral equation, Eq. (9), $\lambda(\varepsilon)$ must have a discontinuity in its first (and therefore also in its second) derivative at $\varepsilon=u$. This is reminiscent of what happens in Lindhard's equation at $\varepsilon=0$, as mentioned at the end of Sec. II. Defining these discontinuities as

$$
\begin{array}{ll}
\lim _{\zeta \rightarrow 0} \lambda^{\prime}(u+\zeta)=\alpha_{1}, & \lim _{\zeta \rightarrow 0} \lambda^{\prime \prime}(u+\zeta)=\alpha_{2}, \\
\lim _{\zeta \rightarrow 0} \lambda^{\prime}(u-\zeta)=0, & \lim _{\zeta \rightarrow 0} \lambda^{\prime \prime}(u-\zeta)=0,
\end{array}
$$

with $\alpha_{1} \neq 0$ and $\alpha_{2} \neq 0$, consistently the condition to make the 1.h.s. in Eq. (9) vanish at $\varepsilon=u$ is given by

$$
\alpha_{1}=1+\frac{1}{2} u \alpha_{2}
$$

Therefore it is only necessary to determine one of the two parameters (e.g., $\left.\alpha_{2}\right)$. In order for $\bar{\nu}(\varepsilon)$ to remain physical, its second and first derivatives must satisfy the conditions

$$
\begin{gathered}
\lim _{\varepsilon \rightarrow \infty} \bar{\nu}^{\prime \prime}(\varepsilon)=0^{-} \text {(from below), and } \\
0 \leq \bar{\nu}^{\prime}(\varepsilon) \leq 1 \quad \text { for } \varepsilon \geq u
\end{gathered}
$$

otherwise $\bar{\nu}$ will not match Lindhard's solution at high energies, if Eq. (18) is not satisfied, or the QF could become, either negative or greater than 1, if Eq. (19) is not satisfied.

For $\varepsilon=u$ the first condition, Eq. (18), restricts the possible values of $\alpha_{2}$ to lie in the interval

$$
-2 / u \leq \alpha_{2} \leq 0
$$

Given $u \neq 0$, and small values of the step size $h$, and tolerance $\delta$, (both $\ll 1)$, we find a solution to Eq. (9) in the interval $u \leq \varepsilon \leq \varepsilon^{\max }$ by means of the following shooting method: 
(1) Set $\varepsilon^{\max }$ to a large initial value $\varepsilon_{0}^{\max }=500 u$, and the limits $\alpha_{2}^{\text {lo }}=-2 / u$, and $\alpha_{2}^{\text {hi }}=0$.

(2) Sample a random value of $\alpha_{2}$ in the interval $\alpha_{2}^{\text {lo }} \leq \alpha_{2} \leq \alpha_{2}^{\text {hi }}$, calculate the corresponding value of $\alpha_{1}$ from Eq. (17), and set the starting values

$$
\varepsilon_{t}=u, \quad \lambda(u)=0, \quad \lambda^{\prime}(u)=\alpha_{1}, \quad \lambda^{\prime \prime}(u)=\alpha_{2} .
$$

(3) If $\varepsilon_{t}=\varepsilon^{\text {max }}$, skip to step 8. Else, use Eq. (15) to calculate $\bar{\nu}\left(\varepsilon_{t}\right), \bar{\nu}^{\prime}\left(\varepsilon_{t}\right)$, and $\bar{\nu}^{\prime \prime}\left(\varepsilon_{t}\right)$.

(4) If the condition in Eq. (19) is satisfied, continue. Else, if it fails because $\bar{\nu}^{\prime}\left(\varepsilon_{t}\right)<0$, set $\alpha_{2}^{\text {hi }}=\alpha_{2}$, and return to 2. Else, if it fails because $\bar{\nu}^{\prime}\left(\varepsilon_{t}\right)>1$, set $\alpha_{2}^{\text {lo }}=\alpha_{2}$, and return to 2 .

(5) Calculate $\lambda\left(\varepsilon_{t}+h\right)$ and $\lambda^{\prime}\left(\varepsilon_{t}+h\right)$ using a second order expansion of $\lambda$ about $\varepsilon_{t}$

$$
\begin{aligned}
\lambda\left(\varepsilon_{t}+h\right) & \approx \lambda\left(\varepsilon_{t}\right)+\lambda^{\prime}\left(\varepsilon_{t}\right) h+\frac{1}{2} \lambda^{\prime \prime}\left(\varepsilon_{t}\right) h^{2}, \\
\lambda^{\prime}\left(\varepsilon_{t}+h\right) & \approx \lambda^{\prime}\left(\varepsilon_{t}\right)+\lambda^{\prime \prime}\left(\varepsilon_{t}\right) h,
\end{aligned}
$$

and calculate $\bar{\nu}\left(\varepsilon_{t}+h\right)$ and $\bar{\nu}^{\prime}\left(\varepsilon_{t}+h\right)$.

(6) Use Eq. (9) to solve for $\bar{\nu}^{\prime \prime}\left(\varepsilon_{t}+h\right)$, evaluating the integral in the r.h.s. numerically by interpolating the behavior of $\bar{\nu}(\varepsilon)$ between $u$ and $\varepsilon_{t}+h$ with cubic splines passing through all previous points.

(7) Set $\varepsilon_{t}$ to $\varepsilon_{t}+h$ and return to 3 .

(8) If the second derivative condition in Eq. (18) at $\varepsilon^{\max }$ is satisfied within a tolerance $\delta$, stop. Else, increment $\varepsilon^{\max }=\varepsilon^{\max }+\Delta$ and return to 2 .

An example of the application of this method to the case of $\mathrm{Si}$ with $u=3.7 \times 10^{-3}$, and 1000 steps uniformly spaced in logarithmic scale in the interval $150 \mathrm{eV}<E_{r}<$ $100 \mathrm{keV}$ is illustrated in Fig. 3. The second derivative condition in Eq. (18) is well satisfied at $\varepsilon$ corresponding to $100 \mathrm{keV}$, although for some values of $u$ and $k$, the condition is satisfied at lower energies, for those cases Eq. (9) in step 6 can be used without the second derivative term.

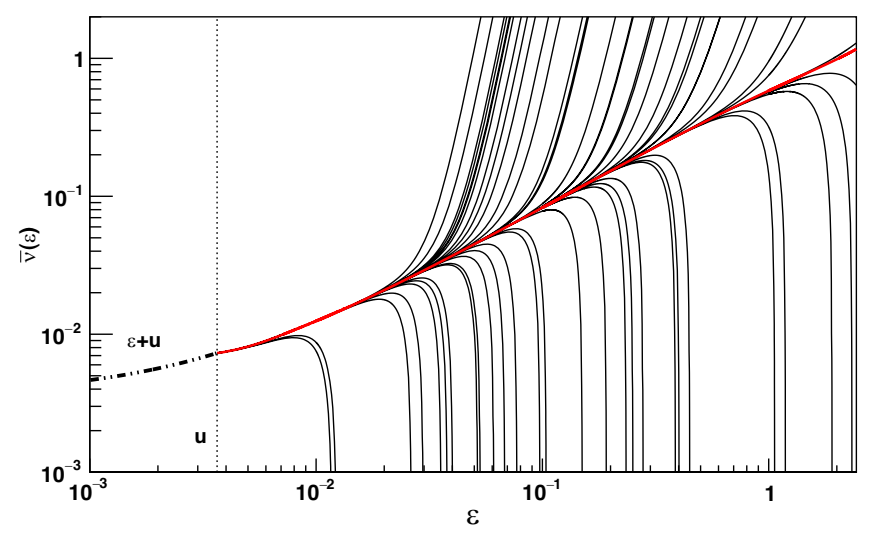

FIG. 3. Shooting method for Si. The red curve is the only one satisfying the boundary conditions in Eqs. (19) and (18).
The solutions from 61 random shots failing to satisfy the conditions in Eqs. (19) and (18) are shown as the black curves. The successful final shot satisfying the conditions in the interval of interest is shown in red.

\section{FITS TO DATA}

The QF data sets used in this study are summarized in Table II. For Si, four data sets have been considered: Zech [31], with 8 points in the energy range from 4.30 to $53.7 \mathrm{keV}$; Brian [32], with 4 points in the energy range from 4.15 to $75.7 \mathrm{keV}$; CHICAGO [2] with 12 points in the energy range from 0.68 to $2.28 \mathrm{keV}$; ANTONELLA [3] with 14 points in the energy range from 1.79 to $20.67 \mathrm{keV}$. The last two are the lowest energy measurements available to date. For Ge, six data sets have been considered: Jones (75) [7], with 1 point at $0.254 \mathrm{keV}$; COGENT [33] with 4 points in the energy range from 0.65 to $1.22 \mathrm{keV}$; TEXONO [9] with 3 points in the energy range from 1.25 to $3.61 \mathrm{keV}$; Messous [34] with 3 points in the energy range from 2.71 to $8.72 \mathrm{keV}$; Shutt [35] with 7 points in the energy range from 17.50 to $70.05 \mathrm{keV}$; Chassman [5] with 16 points in the energy range from 10.04 to $73.17 \mathrm{keV}$.

The ansatz, Eq. (13), with $\varepsilon=\varepsilon_{R}-u$, was fit to the data for each target ion allowing $C_{0}, C_{1}$, and $u$ to vary freely, with the constraint that the QF displays a cutoff in a positive value of $E_{R}$. The numerical solution was also fit to the data varying the parameters $k$ and $u$. The results of the fits are summarized in Table III for the ansatz, and Table IV for the numerical solution, as well as in Fig. 4 for Si and Fig. 5 for Ge. Additionally, we tabulated the numerical solution for the function $f_{n}$ against the recoil energy for $\mathrm{Si}$ and $\mathrm{Ge}$ in Supplemental Tables I and II [36]. The top panel in these figures shows the error calculated using Eq. (14) for the ansatz, and compares it with the error for Lindhard's model tested against his original integral equation, Eq. (5). By construction the error of the numerical solution is negligible $(<0.5 \%)$ and is not shown.

TABLE II. Summary of the data sets used in this study.

\begin{tabular}{lcr}
\hline \hline Data set & Energy range $(\mathrm{keV})$ & \# points \\
\hline & Silicon & \\
Zech [31] & $4.30-53.7$ & 8 \\
Brian [32] & $4.15-75.7$ & 4 \\
CHICAGO [2] & $0.68-2.28$ & 12 \\
ANTONELLA [3] & $1.79-20.67$ & 14 \\
& Germanium & \\
Jones (75) [7] & 0.254 & 1 \\
COGENT [33] & $0.65-1.22$ & 4 \\
TEXONO [9] & $1.25-3.61$ & 3 \\
Messous [34] & $2.71-8.72$ & 3 \\
Shutt [35] & $17.50-70.05$ & 7 \\
Chassman [5] & $10.04-73.17$ & 16 \\
\hline \hline
\end{tabular}


TABLE III. Fitted parameters for the ansatz in Eq. (13) for the different data sets. We report the binding energy $U=u / c_{Z}$. High $\chi^{2} /$ ndf reflect the tension among the data sets given the reported errors. The uncertainties are estimated so as to cover the variations among the data sets.

\begin{tabular}{lcccc}
\hline \hline & $C_{0}$ & $C_{1}\left(\times 10^{-5}\right)$ & $U(\mathrm{keV})$ & $\chi^{2} / \mathrm{ndf}$ \\
\hline $\mathrm{Si}$ & $(9.1 \pm 4.4) \times 10^{-3}$ & $3.33 \pm 1.2$ & $0.15 \pm 0.06$ & $224 / 40$ \\
$\mathrm{Ge}$ & $(3.0 \pm 1.3) \times 10^{-4}$ & $0.62 \pm 0.12$ & $0.02 \pm 0.01$ & $56 / 35$ \\
\hline \hline
\end{tabular}

TABLE IV. Fitted parameters for the numerical solution to the different data sets. We report the binding energy $U=u / c_{Z}$. High $\chi^{2} /$ ndf reflect the tension among the data sets given the reported errors. The uncertainties are estimated so as to cover the variations among the data sets.

\begin{tabular}{lccc}
\hline \hline & $k$ & $U(\mathrm{keV})$ & $\chi^{2} / \mathrm{ndf}$ \\
\hline $\mathrm{Si}$ & $0.161_{-0.020}^{+0.029}$ & $0.15_{-0.05}^{+0.10}$ & $349.2 / 40$ \\
$\mathrm{Ge}$ & $0.162_{-0.021}^{+0.028}$ & $0.02_{-0.010}^{+0.015}$ & $52.3 / 35$ \\
\hline \hline
\end{tabular}

The fits of the ansatz and the numerical solution give high values of $\chi^{2}$ per degree of freedom for $\mathrm{Si}$ and $\mathrm{Ge}$, which are indicative of the tension among the different data sets. The uncertainties that we report in Tables III and IV were estimated so as to approximately cover the variation among the different measurements, and in the case of Xe, to cover the large uncertainties reported. This is shown in the error bands in Figs. 4 and 5.

For Si data, the ansatz fit (see Table III) gives a value of the binding energy of $U=0.15 \pm 0.06 \mathrm{keV}$, while the fit of the numerical solution (see Table IV) gives $k=0.145_{-0.020}^{+0.029}$, and $U=0.15_{-0.05}^{+0.10} \mathrm{keV}$. The fitted value of $k$ is well within the expected values extracted from the older data in the range from $10-100 \mathrm{keV}$ fitted to Lindhard's model. On the other hand, the fitted binding
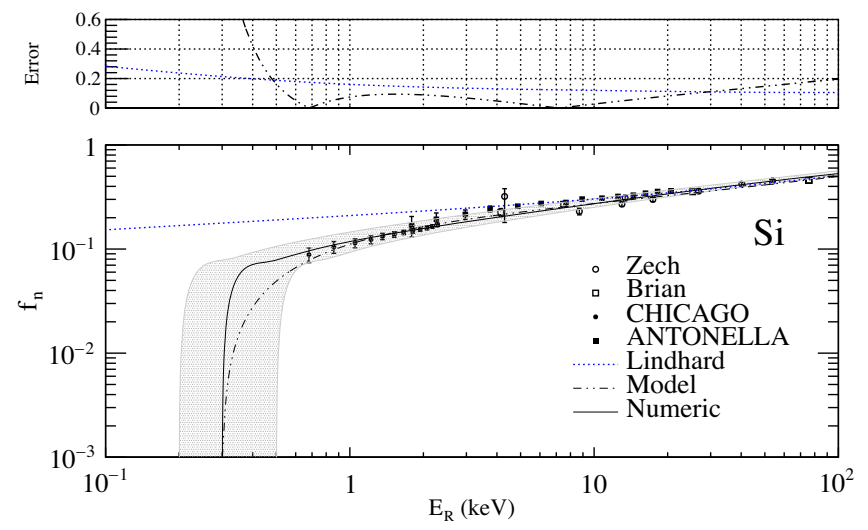

FIG. 4. (Lower panel) Measurements of the QF in Si (points with error bars) compared to the Lindhard model (dot-dashed line), the ansatz of Eq. (13), and the numerical solution with $U=0.15 \mathrm{keV}$ and $k=0.161$. (Upper panel) Error in the ansatz and the Lindhard original model.

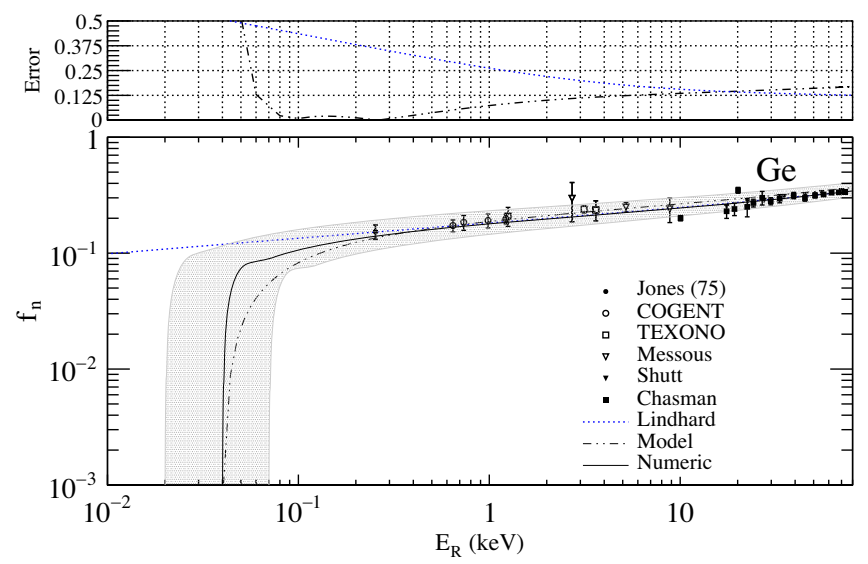

FIG. 5. (Lower panel) Measurements of the QF in Ge (points with error bars) compared to the Lindhard model (dot-dashed line), the fitted ansatz of Eq. (13), and the numerical solution with $U=0.02 \mathrm{keV}$ and $k=0.162$. (Upper panel) Error in the ansatz and the Lindhard original model.

energy is consistent with a picture where the recoiling ion causes, on average, the ionization of one electron from the $2 p$ shell, as well as the creation of several $e-h$ pairs and Frenkel pair defects. The cutoff of the QF at $E_{r} \approx 300 \mathrm{eV}$ is an artifact of the constant $u$ model arising from the relatively high value of the binding energy, compared to the energy required to produce $e-h$ pairs or lattice defects in Si, which limits the applicability of the model to $E_{r} \gtrsim 500 \mathrm{eV}$.

For the Ge data, the ansatz fit gives a value of $U=0.02 \pm 0.01 \mathrm{keV}$, and the fitted numerical solution gives $k=0.188_{-0.024}^{+0.017}$, and $U=0.02_{-0.010}^{+0.015} \mathrm{keV}$. Once more, the fitted value of $k$ agrees well with previous estimates, since the available data can be described reasonably well by Lindhard's original model. Interestingly, since in this case the binding energy is of the same order of magnitude as the energy required to create lattice defects, a naive picture can be considered. The recoiling ion can cause, either the ionization of one electron from the $3 d$ shell, as well as a few $e-h$ pairs, or instead, the creation of one Frenkel-pair and several $e-h$ pairs. The cutoff of the QF from the numerical solution appears at $E_{r} \approx 40 \mathrm{eV}$, which is likely closer to the physical threshold for this target atom. In this case, our constant $u$ model is expected to give a reasonable description all the way down to recoil energies of $E_{r} \gtrsim 50 \mathrm{eV}$, much closer to the physical threshold, which can be safely expected to lie somewhere between a few $\mathrm{eV}$ and a few tens of eV.

Although the ansatz gives a reasonable description of the data, the numerical solution does so too using only two parameters, and is therefore preferred. Figure 6 shows a comparison of the numerical solutions obtained for the three targets considered in this work. In this figure, we have modified the numerical solution for $\mathrm{Si}$ to provide a good match to the data below $40 \mathrm{keV}$, which follows very closely 


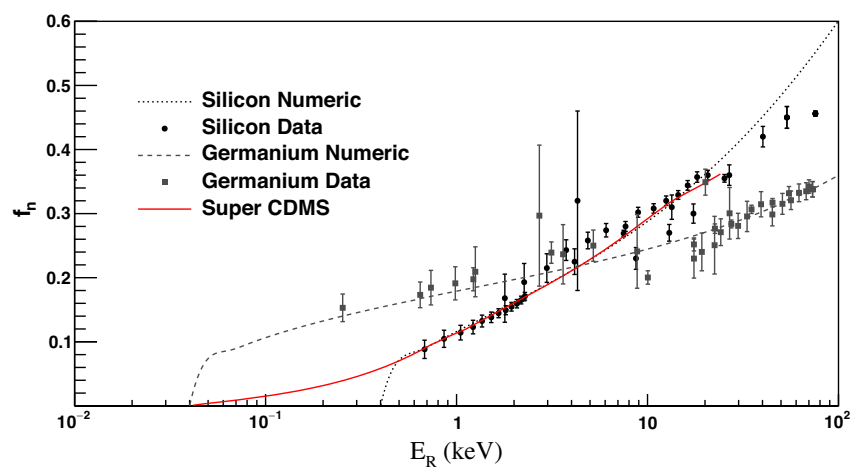

FIG. 6. Comparison of the numerical solutions for $\mathrm{Si}, \mathrm{Ge}$, with data. The Si curve has been changed from that in Fig. 4 to fit only the data $<40 \mathrm{keV}$ ( $k=0.169$ and $U=0.2 \mathrm{keV}$ ). Also shown is the phenomenological fit by Super-CDMS [37] (red solid line).

the phenomenological fit reported by the Super-CDMS Collaboration [14], shown in the solid red line in the figure. The three Si measurements above this energy are likely affected by nuclear charge screening effects, as is suggested by the change in behavior already seen in the SuperCDMS fit.

\section{CONCLUSIONS}

We found an appropriate form for the basic integrodifferential equation describing the energy given to atomic motion by nuclear recoils in a homogeneous medium, such as pure crystals, when the binding energy is taken into account. Assuming a constant average binding energy, $u \neq 0$, we give approximate semianalytical solutions, motivated by the analysis of the integro-differential equation, that are in reasonable agreement with the available experimental measuremens of the QF for nuclear recoils in $\mathrm{Si}$ and Ge. Numerical solutions depending only on the constant binding energy and the electronic stopping power factor $k$ were calculated and found to be also consistent with the data. As expected, our solutions for the QF display a cutoff at a value equal to twice the binding energy, $2 u$. This cutoff is a feature due to the threshold of the cascading process built into the model.

Measurements of QF in Ge detectors are well described by the our model, with $k$ within the expected range $(0.1<k<0.2)$. We predict that the QF cutoff in this material is in the range between $20-70 \mathrm{eV}$ of nuclear recoil energy, corresponding to a binding energy of $10-35 \mathrm{eV}$. The Frenkel pair dislocation energy in Ge falls well within this interval, and is expected to be an upper limit close to the physical cutoff, believed to be of the order of only a few $\mathrm{eV}$. In a more realistic scenario, where the ion is only required to acquire sufficient motion to generate phonon excitations that can then take an electron from the valence to the conduction band, such a low physical cutoff could be explained.
In the case of $\mathrm{Si}$, the $\mathrm{QF}$ measurements are well described by our model with $k$ within the expected limits, only if the binding energy is in the range $100-250 \mathrm{eV}$. Now, the predicted cutoff is much larger than the Frenkel energy of about $36 \mathrm{eV}$, and therefore also greater than the physical cutoff. Hence, the model should be valid only for nuclear recoil energies above $500 \mathrm{eV}$. A more accurate description, considering the variation of the binding energy and stopping power with the recoiling ion energy could be considered. In addition, effects appearing at higher energies, such as ion charge screening (e.g., Bohr stripping [38]) could manifest as a change in the value of $k$ at recoil energies of a few tens of $\mathrm{keV}$.

In summary, the model described here, depending only on a constant binding energy and a slope of the velocity-proportional electronic energy loss in the range $0.1<k<0.2$, can explain the behavior of the QF measured to date in pure element targets of $\mathrm{Si}$ and $\mathrm{Ge}$. We expect the model to give a reasonable approximation to the physical cutoff in cases where the binding energy is lower than or comparable to the Frenkel-pair energy, as it is the case for Ge.

\section{ACKNOWLEDGMENTS}

This research was supported in part by DGAPA-UNAM Grant No. PAPIIT-IN108917, and Consejo Nacional de Ciencia y Tecnología (CONACYT) through Grant No. CB2014/240666. The authors wish to thank Guillermo Fernandez Moroni and Juan Estrada for useful discussions.

\section{APPENDIX A: SEMIHARD SPHERES COLLISION}

The semihard sphere model can be used to calculate the minimum scattering angle, and hence the minimum value of $t$. Taking into account the binding energy $u$, the total energy is $\varepsilon+v$, where $v$ is

$$
v= \begin{cases}0 & \text { for } \mathrm{r} \in[R, \infty] \\ -u & \text { for } \mathrm{r} \in\left[R_{0}, R\right] \\ \infty & \text { for } \mathrm{r} \in\left[0, R_{0}\right]\end{cases}
$$

In order to estimate the minimum scattering angle for this scenario we use as an approximation the classical formula for the scattering angle from a potential

$$
\theta_{\min }=\pi-2 b \int_{r_{\min }}^{\infty} \frac{d r}{r^{2} \sqrt{1-(b / r)^{2}-v /(\varepsilon+v)}},
$$

where $b$ is the impact parameter (set to $R_{0}$, as shown in Fig. 7), $r_{\min }$ is the turning point of the potential, and $v$ is given in Eq. (A1).

For the potential in Eq. (A1) we can split the integral (A2) in three parts: one from zero to $R_{0}$, another form $R_{0}$ to $R$, and the third from $R$ to $\infty$. The first integral is zero, so the minimum angle is given by 


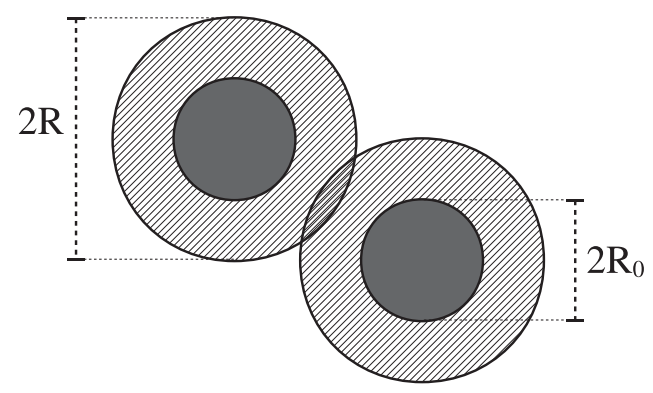

FIG. 7. Diagram of a collision between two semihard spheres.

$$
\begin{aligned}
\theta_{\min }= & \pi-\int_{R_{0}}^{R} \frac{2 R_{0} d r}{r^{2} \sqrt{1-\left(R_{0} / r\right)^{2}+u /(\varepsilon-u)}} \\
& -\int_{R}^{\infty} \frac{2 R_{0} d r}{r^{2} \sqrt{1-\left(R_{0} / r\right)^{2}}},
\end{aligned}
$$

Assuming that $R_{0} \propto a_{0} / Z$, where $a_{0}$ is the Bohr radius and $R \propto 2 a_{0}$, for $Z>5$ we have $R \gg R_{0}$, so we can approximate Eq. (A3) by

$$
\theta_{\min } \cong \pi-\int_{R_{0}}^{\infty} \frac{2 R_{0} d r}{r^{2} \sqrt{1-\left(R_{0} / r\right)^{2}+u /(\varepsilon-u)}} .
$$

Calculating the integral (A4) we arrive at

$$
\sin ^{2}\left(\theta_{\min } / 2\right)=\frac{u}{\varepsilon}
$$

which in terms of the variable $t$ has a minimum at $t_{\min }=u \varepsilon$, as is used in Eq. (9). The same result can be derived from the model in Ref. [39] (page 131) adapted to the collision of semihard spheres.

\section{APPENDIX B: SECOND ORDER TERM IN THE MODIFIED SIMPLIFIED INTEGRAL EQUATION}

Substitution of Eq. (8) in Eq. (3) and integration over the nuclear and electronic cross sections, putting also in effect approximation (D), leads to the appearance of the electronic stopping power

$$
\begin{aligned}
\int d \sigma_{n, e} \bar{\nu}^{\prime}(E)\left(\Sigma_{i} T_{e i}\right) & =\nu^{\prime}(E) \int d \sigma_{e}\left(\Sigma_{i} T_{e i}\right) \\
& \propto \nu^{\prime}(\varepsilon) S_{e}(\varepsilon)
\end{aligned}
$$

in the first derivative term, as in the original formulation by Lindhard. In the second derivative term, we can apply the integral mean value theorem to write

$$
\begin{aligned}
\int d \sigma_{n, e} \bar{\nu}^{\prime \prime}(E) T_{n}\left(\Sigma_{i} T_{e i}\right) & =\nu^{\prime \prime}(E) \bar{T}_{n} \int d \sigma_{e}\left(\Sigma_{i} T_{e i}\right) \\
& \propto \nu^{\prime \prime}(\varepsilon) \bar{t}_{n} S_{e}(\varepsilon),
\end{aligned}
$$

where $\bar{t}_{n}=c \bar{T}_{n}$ is a suitable average value of the energy transfer $t_{n}=\varepsilon \sin ^{2}(\theta / 2)$, which we will approximate by $\bar{t}_{n} \approx\left\langle t_{n}\right\rangle=\frac{1}{2} \varepsilon$, leading to the final form of our proposed modified simplified integral equation Eq. (9).
[1] J. Lindhard, V. Nielsen, M. Scharff, and P. Thomsen, Integral equations governing radiation effects, Kong. Dan. Vid. Sel. Mat. Fys. Med. 33 (1963), http://www.sdu.dk/ Bibliotek/matfys.

[2] A. E. Chavarria et al., Measurement of the ionization produced by sub-keV silicon nuclear recoils in a CCD dark matter detector, Phys. Rev. D 94, 082007 (2016).

[3] F. Izraelevitch et al., A measurement of the ionization efficiency of nuclear recoils in silicon, J. Instrum. 12, P06014 (2017).

[4] P. S. Barbeau, J. I. Collar, and O. Tench, Large-mass ultralow noise germanium detectors: Performance and applications in neutrino and astroparticle physics, J. Cosmol. Astropart. Phys. 09 (2007) 009.

[5] C. Chasman, K. W. Jones, and R. A. Ristinen, Measurement of the Energy Loss of Germanium Atoms to Electrons in Germanium at Energies Below $100 \mathrm{keV}$, Phys. Rev. Lett. 15, 245 (1965).

[6] C. Chasman, K. W. Jones, H. W. Kraner, and W. Brandt, Band-Gap Effects in the Stopping of Ge-72* Atoms in Germanium, Phys. Rev. Lett. 21, 1430 (1968).
[7] K. W. Jones and H. W. Kraner, Energy lost to ionization by 254-eV Ge-73 atoms stopping in Ge, Phys. Rev. A 11, 1347 (1975).

[8] Y. Messous, Calibration of a Ge crystal with nuclear recoils for the development of a dark matter detector, Astropart. Phys. 3, 361 (1995).

[9] B. J. Scholz, A. E. Chavarria, J. I. Collar, P. Privitera, and A. E. Robinson, Measurement of the low-energy quenching factor in germanium using an ${ }^{88} \mathrm{Y} / \mathrm{Be}$ photoneutron source, Phys. Rev. D 94, 122003 (2016).

[10] P. Sorensen, Atomic limits in the search for galactic dark matter, Phys. Rev. D 91, 083509 (2015).

[11] J. Gascon, Journées matière sombre france, France (2017), https://indico.in2p3.fr/event/16464/.

[12] C. E. Aalseth et al. (CoGeNT Collaboration), Results from a search for Light-Mass Dark Matter with a $p$-Type Point Contact Germanium Detector, Phys. Rev. Lett. 106, 131301 (2011).

[13] R. Agnese et al. (SuperCDMS Collaboration), Search for low-mass dark matter with cdmslite using a profile likelihood fit, Phys. Rev. D 99, 062001 (2019). 
[14] R. Agnese and E. A. Anderson (SuperCDMS Collaboration), Projected sensitivity of the supercdms snolab experiment, Phys. Rev. D 95, 082002 (2017).

[15] Aguilar-Arevalo et al. (DAMIC Collaboration), Search for low-mass wimps in a $0.6 \mathrm{~kg}$ day exposure of the damic experiment at snolab, Phys. Rev. D 94, 082006 (2016).

[16] N. Castelló-Mor (DAMIC-M Collaboration), Damic-m experiment: Thick, silicon ccds to search for light dark matter, Nucl. Instrum. Methods Phys. Res., Sect. A 958, 162933 (2019).

[17] A. Aguilar-Arevalo et al. (CONNIE Collaboration), Exploring low-energy neutrino physics with the coherent neutrino nucleus interaction experiment, Phys. Rev. D 100, 092005 (2019).

[18] B. C. F. K. E. A. Hakenmüller, Neutron-induced background in the conus experiment, Eur. Phys. J. C 79, 699 (2019).

[19] H. T.-K. Wong, Taiwan experiment on neutrino-history and prospects, Int. J. Mod. Phys. A 33, 1830014 (2018).

[20] A. B. Migdal, Ionization of atoms accompanying $\alpha$ and $\beta$ decay, J. Phys. (USSR), Lett. 4, 449 (1941).

[21] G. Baur, F. Rosel, and D. Trautmann, Ionisation induced by neutrons, J. Phys. B 16, L419 (1983).

[22] E. Fermi, Eine statistische methode zur bestimmung einiger eigenschaften des atoms und ihre anwendung auf die theorie des periodischen systems der elemente, Z. Phys. 48, 73 (1928).

[23] W. D. Wilson, L. G. Haggmark, and J. P. Biersack, Calculations of nuclear stopping, ranges, and straggling in the low-energy region, Phys. Rev. B 15, 2458 (1977).

[24] A. A. Correa, Calculating electronic stopping power in materials from first principles, Comput. Mater. Sci. 150, 291 (2018).

[25] J. F. Ziegler and J. P. Biersack, The stopping and range of ions in matter, in Treatise on Heavy-Ion Science: Volume 6: Astrophysics, Chemistry, and Condensed Matter, edited by D. A. Bromley (Springer US, Boston, MA, 1985), pp. 93-129.

[26] K. B. Winterbon, P. Sigmund, and J. B. Sanders, Spatial distribution of energy deposited by atomic particles in elastic collisions, K. Dan. Vidensk. Selsk. 37, 14 (1970), https://www.osti.gov/biblio/4076056.

[27] E. Holmström, A. Kuronen, and K. Nordlund, Threshold defect production in silicon determined by density functional theory molecular dynamics simulations, Phys. Rev. B 78, 045202 (2008).

[28] E. Holmström, K. Nordlund, and A. Kuronen, Threshold defect production in germanium determined by density functional theory molecular dynamics simulations, Phys. Scr. 81, 035601 (2010).

[29] J. A. Bearden and A. F. Burr, Reevaluation of x-ray atomic energy levels, Rev. Mod. Phys. 39, 125 (1967).

[30] M. Cardona and E. L. Ley, Photoemission in Solids $i$ : General Principles (Springer-Verlag, Berlin, 1978).

[31] P. Zecher, D. Wang, J. Rapaport, C. J. Martoff, and B. A. Young, Energy deposition of energetic silicon atoms within a silicon lattice, Phys. Rev. A 41, 4058 (1990).

[32] B. L. Dougherty, Measurements of ionization produced in silicon crystals by low-energy silicon atoms, Phys. Rev. A 45, 2104 (1992).

[33] C. E. Aalseth et al. (CoGeNT Collaboration), Cogent: A search for low-mass dark matter using $p$-type point contact germanium detectors, Phys. Rev. D 88, 012002 (2013).

[34] Y. Messous et al., Calibration of a ge crystal with nuclear recoils for the development of a dark matter detector, Astropart. Phys. 3, 361 (1995).

[35] T. Shutt et al., Measurement of Ionization and Phonon Production by Nuclear Recoils in a $60 \mathrm{~g}$ Crystal of Germanium at 25 mk, Phys. Rev. Lett. 69, 3425 (1992).

[36] See Supplemental Material at http://link.aps.org/ supplemental/10.1103/PhysRevD.101.102001, for the quenching factor values as a function of nuclear recoil energy $\left(E_{R}\right)$ for silicon, and germanium, respectively, as obtained from the numerical solution to Eq. (9), described in Secs. III and IV. The values correspond to the solid black line in Fig. 4 (Si) and Fig. 5 (Ge).

[37] R. Agnese et al. (SuperCDMS Collaboration), Projected sensitivity of the supercdms snolab experiment, Phys. Rev. D 95, 082002 (2017).

[38] M. Ç. Tufan, A. Köroğlu, and H. Gümüş, Stopping power calculations for partially stripped projectiles in high energy region, Acta Phys. Pol. A 107, 459 (2005).

[39] V. Zelevinsky, Quantum Physics Vol. 2 (John Wiley \& Sons, New York, 2010). 